\title{
BMJ Open Factors associated with non-persistence to oral and inhaled antiviral therapies for seasonal influenza: a secondary analysis of a double-blind, multicentre, randomised clinical trial
}

\author{
Remi Flicoteaux, ${ }^{1,2}$ Camelia Protopopescu, ${ }^{3,4}$ Annick Tibi, ${ }^{5}$ Thierry Blanchon, ${ }^{6}$ \\ Sylvie Van Der Werf, ${ }^{7}$ Xavier Duval, ${ }^{1}$ Anne Mosnier, ${ }^{8}$ Cécile Charlois-Ou, ${ }^{1}$ \\ Bruno Lina, ${ }^{9,10}$ Catherine Leport, ${ }^{1}$ Sylvie Chevret ${ }^{2}$
}

To cite: Flicoteaux R, Protopopescu C, Tibi A, et al. Factors associated with non-persistence to oral and inhaled antiviral therapies for seasonal influenza: a secondary analysis of a double-blind, multicentre, randomised clinical trial. BMJ Open 2017;7:e014546. doi:10.1136/ bmjopen-2016-014546

- Prepublication history and additional material are available. To view these files please visit the journal online (http://dx.doi. org/10.1136/bmjopen-2016014546).

Received 7 0ctober 2016 Revised 10 May 2017 Accepted 1 June 2017

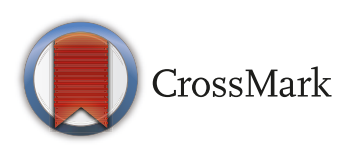

For numbered affiliations see end of article.

Correspondence to Remi Flicoteaux; remi. flicoteaux@aphp.fr

\section{ABSTRACT}

Objectives We aimed to evaluate and compare nonadherence to oral and inhaled antiviral therapies prescribed of a randomised clinical trial in outpatients with influenza A infection.

Design A parallel, three-arm, double-blinded trial randomly allocated antiviral therapies twice daily for 5 days: (1) oral oseltamivir plus inhaled zanamivir (arm 0Z); (2) oseltamivir plus inhaled placebo (arm Opz); or (3) oral placebo plus inhaled zanamivir (arm poz). Analysis of nonadherence was a secondary objective of the trial.

Settings Outpatients were enrolled by 145 general practitioners throughout France during the 2008-2009 seasonal influenza epidemics.

Participants A total of 541 adults presenting with influenza-like illness for less than 36 hours.

Primary outcomes Non-persistence, the time between inclusion and the last dose treated as a failure time, was used as the primary endpoint.

Results The proportions of patients who persisted on treatment until the end of prescription were estimated at $85.73 \%( \pm 3.28 \%)$ for the oral route and $82.73 \%( \pm 3.44 \%)$ for the inhaled route. Based on multivariable models, nonpersistence was associated with a PCR confirmation of influenza for both the oral ( $\mathrm{HR}=0.54, \mathrm{p}=0.010)$ and inhaled ( $H R=0.59, p=0.018)$ drugs and antibiotic coprescriptions ( $\mathrm{HR}=2.07, \mathrm{p}=0.007$; and $\mathrm{HR}=1.88, \mathrm{p}=0.017$, respectively) and active combination treatment $(H R=1.71, p=0.035$; and $H R=1.58, p=0.035$, respectively). The hazard of nonpersistence of the inhaled therapy was increased compared with that of the oral therapy ( $\mathrm{HR}=1.23, \mathrm{p}=0.043)$.

Conclusion In addition to the clinical and virological profiles of influenza infection, non-persistence may have been influenced by an active combination and the route of administration.

RCT registration number NCT00799760. This is a postresult analysis.

\section{INTRODUCTION}

The increase in drug-resistant influenza viruses, both epidemic and pandemic strains, is a major public health concern.
Strengths and limitations of this study

- We provide a precise description of longitudinal treatment dropout and loss to follow-up from a double-blind randomised clinical trial to assess the efficacy of the combination of oseltamivir plus zanamivir compared with each monotherapy for influenza.

- We used a definition from the recent taxonomy of non-adherence that splits this complex outcome into two main components: persistence and execution.

- We used specific methods to address missing data and loss to follow-up, a constant issue in studies assessing the efficacy of outpatient treatments.

- The measurement of patients' dosing regimen history was not performed via recommended electronic monitoring but by combining self-reported diaries and pill counts.

Moreover, meta-analysis has questioned the efficacy of antiviral monotherapies. ${ }^{1}$ The use of combined therapy has been advocated as a solution to this issue. Therefore, a doubleblind randomised clinical trial (RCT) was conducted among 541 patients with seasonal influenza A (mainly H3N2 viral infection) to assess the clinical and virological responses to a combination of oral oseltamivir and inhaled zanamivir compared with each monotherapy combined with a placebo of the other drug. The results of this trial (BIVIR, NCT00799760), based on an intent-to-treat analysis, showed that for treating seasonal influenza, the oseltamivir-zanamivir combination was less effective than oseltamivir monotherapy and was not significantly more effective than zanamivir monotherapy. ${ }^{2}$

One important factor of successful therapy is adherence to the prescribed medicine, 
notably among ambulatory patients, such as patients with influenza. ${ }^{3}$ Adherence is defined as 'the extent to which a person's behaviour-taking medication, following a diet, and/or executing lifestyle changes-corresponds with agreed recommendations from a health care provider'. ${ }^{4}$ Medication adherence (with patient compliance) is the most commonly used metric to describe deviations from prescribed dosing regimens. ${ }^{3}$ However, if non-adherence affects the outcome of therapy, it might also be a major determinant in the emergence of viral resistance, particularly in the context of antiviral therapies. Indeed, low drug concentrations might not inhibit viral replication, and this replication in the presence of antiviral drugs promotes the emergence of viral resistance. In addition, low adherence may interfere with the intent-to-treat analysis of clinical trials, which is currently a point of particular attention. ${ }^{5}$ Notably, as reported by Heneghan et al, ${ }^{6}$ this issue is very common in RCTs evaluating the efficacy of antiviral therapies for influenza disease. Indeed, in their meta-analysis, none of the included studies reported data on the complete follow-up of patients. Thus, a precise description of non-adherence may guide the use of alternative analysis strategies, ${ }^{7-9}$ and as such defined a secondary objective of the BIVIR trial.

Thus, we conducted analyses of the BIVIR trial data to (1) describe patient non-adherence to the combination therapy, (2) compare patient non-adherence to the oral and inhaled therapies and (3) investigate potential factors of non-adherence.

\section{METHODS}

The BIVIR trial is a community-based, double-blind RCT that was conducted between 7 January 2009 and 15 March 2009 (ie, the influenza epidemic period during the winter of that year in France). The methods associated with this trial have been published elsewhere ${ }^{2}$ and are only briefly reported here.

\section{RCT procedures}

A total of 145 general practitioners (GPs) enrolled 541 adult patients who were older than 18 years of age, were within 36 hours of the onset of influenza symptoms and had a positive nasal rapid test for influenza. All of the patients provided written informed consent, and the protocol was approved by the Ethics Committee of Ile de France and was registered in ClinicalTrials.gov (NCT00799760). The treatment efficacy was assessed virologically, on the proportion of patients with nasal influenza reverse transcription (RT)-PCR levels below 200 copies per genome at day 2 (primary outcome), as well as clinically by the time to alleviation of symptoms. ${ }^{2}$

The patients were allocated to one of the following three study arms: (1) oral oseltamivir $75 \mathrm{mg}$ plus inhaled zanamivir $10 \mathrm{mg}(\mathrm{OZ})$; (2) oseltamivir plus inhaled placebo $(\mathrm{Opz})$; or (3) oral placebo plus inhaled zanamivir (poZ). All of the treatments were scheduled to be administered twice daily for 5 days. Patient randomisation was balanced across the three arms and was stratified by the participating GP.

Either a $75 \mathrm{mg}$ oseltamivir pill (Roche, Bale, Switzerland) or its oral placebo was combined with either a $10 \mathrm{mg}$ zanamivir Rotadisk or its inhaled placebo. The commercialised GlaxoSmithKline Diskhaler (GlaxoSmithKline, Philadelphia, Pennsylvania, USA) was used, which required patients to take two inhalations (one $5 \mathrm{mg}$ Rotadisk blister/inhalation for a total of $10 \mathrm{mg}$ ). Once the instructions for capsule intake and Diskhaler use were provided to the patient, the first dose was administered in the presence of the GP. The patients received treatment units that contained the following: (1) a tablet of 10 oseltamivir or placebo pills and (2) a package containing five zanamivir or placebo Rotadisks (total of 10 doses) and 1 Diskhaler inhalation device.

Specific case report forms (CRFs) were completed by the GP. Moreover, patient diaries were given to enrolled patients to collect information on adherence, symptoms and quality of life. The patients were asked to answer questions regarding their intake of the oral and inhaled treatments and the presence of symptoms each time they took a scheduled dose. The severity levels of seven symptoms (ie, nasal stuffiness, sore throat, cough, muscle aches, tiredness or fatigue, headache and feverishness) were rated by the patient twice daily (morning and evening) up to day 5 and then once daily on a 4-point scale ( 0 , none; 1 , mild; 2 , moderate; and 3 , severe). The time to resolution of illness was defined as the time from study drug initiation to the time of symptom alleviation.

The treatment units were collected and analysed by the referent study pharmacist. A pill counts (PC) was performed by the pharmacist when possible.

\section{Description of non-adherence}

Recently adopted taxonomy distinguishing three successive phases of medication adherence, namely initiation, implementation of the dosing regimen and persistence with treatment, was used. ${ }^{3}$ Given that the first dose was administered at the clinical site, which insured treatment initiation, we first focused on implementation by studying treatment execution through the description of dose omissions. Then we described persistence through a time-dependent variable where patients are still on treatment. Omissions followed by dosing were analysed as failures of treatment execution where patients were still considered under treatment (implementation), as opposed to discontinuation (non-persistence) where missing dosing is followed by missing dosing to the rest of the observation period. In this RCT setting, the main study endpoint was defined as the time between inclusion and the last dose treated as a failure of time, that is, the non-persistence. ${ }^{3}$ We computed the number of reported individual treatment intakes for each patient based on patient diaries, the CRF and pill count data. Adherence (implementation and persistence) was studied separately for the oral and inhaled administration routes. 


\section{Statistical methods}

All patients included in the trial were analysed.

To study the execution and to describe the pattern of treatment omissions, as well as of missing data (loss of sights), plots of actually taken doses, omissions and missing data at each time point were drawn, and causes of dropouts were described.

Multiple imputation by chained equations (MICE) was used to impute missing information regarding actual patient dosing ${ }^{10}$; this method incorporated all of the baseline demographic and clinical covariates, the randomisation arm, and sequentially the symptoms and actual doses taken at each time point.

We first used summary statistics, averaged over $\mathrm{M}=50$ imputed data sets, to quantify the implementation of the two oral and inhaled dosing regimens, that is, the proportion of prescribed doses taken over time, for each patient.

Treating persistence as time-to-event data, we then used Kaplan-Meier (KM) curves to estimate the proportion of persistent patients over time; the observation period included 10 points representing each scheduled dose. To show the variability of estimates resulting from the imputation of missing data, we displayed all of the KM curves of all the 50 imputed data sets.

A Cox proportional hazards model was then used to assess factors associated with non-persistence and to compare non-persistence between the two routes of administration, using univariable, then multivariable models. The following potential predictors were considered: age, sex, tobacco use, underlying chronic diseases, influenza A viral load (RT-PCR), confirmed influenza, treatment arm, symptom score on day 0 (randomisation), household composition, occupation and other treatments prescribed. The influence of the oral versus the inhaled route of administration on the hazard of non-persistence was also assessed using robust estimators of variance to handle correlation between the two observations of each patient. Multivariable models considered variables selected from univariable models, although to limit collinearity specialised knowledge of the data allowed us to include only influenza confirmation rather than viral load. Stepwise selection procedure was also performed to check extra useful information of retained predictors. Proportional hazard assumptions were checked and met for all variables. We also tested the effect of disease score, included as a time-dependent variable in the multivariable model. Note that GP effect was assessed through frailty terms, the variance of which did not significantly differ from zero.

Primary analyses used pooled estimates from the 50 MICE data sets. Finally, sensitivity analyses were performed with different assumptions regarding missing data: (1) patients with incomplete information were excluded from the analysis (complete case analysis); (2) all missing data were imputed as 'correct execution' of the treatment; and (3) all missing data were imputed as treatment discontinuations.
Additional analyses using discrete time versions of the proportional hazard model with a complementary log$\log$ link function were performed.

All statistical analyses were performed using $\mathrm{R}$ software (http://www.R-project.org/); the $\mathrm{R}$ package mice was used to perform the multiple imputation. ${ }^{11}$ Two-sided $p$ values less than or equal to 0.05 were considered to be statistically significant.

\section{RESULTS}

Among the 541 patients who were included in the study, a total of 5410 treatment intakes were scheduled for each route of administration. Based on the combination of individual data from the SQ, CRF and PC analyses, the treatment could be considered to be taken, not taken or missing for each of the scheduled treatment intakes (ie, 10 -fold for each route). Details about this combination are provided in the online supplementary materials.

Figure 1 shows the patients' drug histories according to administration route and randomisation arm, illustrating punctual treatment omissions and treatment discontinuation, as well as missing data. Most of the self-reported deviations in medication were driven by treatment discontinuation and, thus, non-persistence. Punctual omissions consisted of one, two or at most three doses not taken. The distribution of missing data presents a similar patterns: punctual missing information and loss to follow-up.

There were $39(7.2 \%)$ and $47(8.7 \%)$ patients who had at least one missing data point during their follow-up for oral and inhaled treatments, respectively. Further results deal with data from multiple imputations unless specified. During implementation of oral treatment, $80 \%$ of patients omitted only one dosing, and $14 \%$ only omitted two or three, while $6 \%$ of patients displayed a more atypical pattern with more than three omissions followed by retreatment. For inhaled treatment, $75 \%$ of patients omitted one single omission, $20 \%$ two or three omissions and $5 \%$ more than three.

Figure 2 (black plots) displays the estimated distribution of time to non-persistence. For oral treatment, the percentages of persistent patients were estimated at $95.6 \%$ $( \pm 1.9 \%)$ at 48 hours and $85.7 \%( \pm 3.3 \%)$ at day 5 (the end of follow-up); for inhaled treatment, the percentages of persistent patients were estimated at $92.9 \%(2.3 \%)$ at 48 hours and $82.7 \%( \pm 3.5 \%)$ at day 5 .

Table 1 reports the univariable analyses of non-persistence predictors. The hazard of non-persistence decreased with the viral load (ie, the higher the viral load, the higher the likelihood of persistence) and, as expected, with having confirmed influenza by PCR, while it increased with the duration of influenza symptoms prior to inclusion in the study (ie, the longer the duration, the higher the likelihood of non-persistence) and with the coprescription of antibiotics. Randomisation also affected non-persistence; patients in the $\mathrm{OZ}$ arm had increased risk of non-persistence, with HRs of $1.71((95 \%$ CI 1.04 to 2.81$), \mathrm{p}=0.022)$ for the oral route 

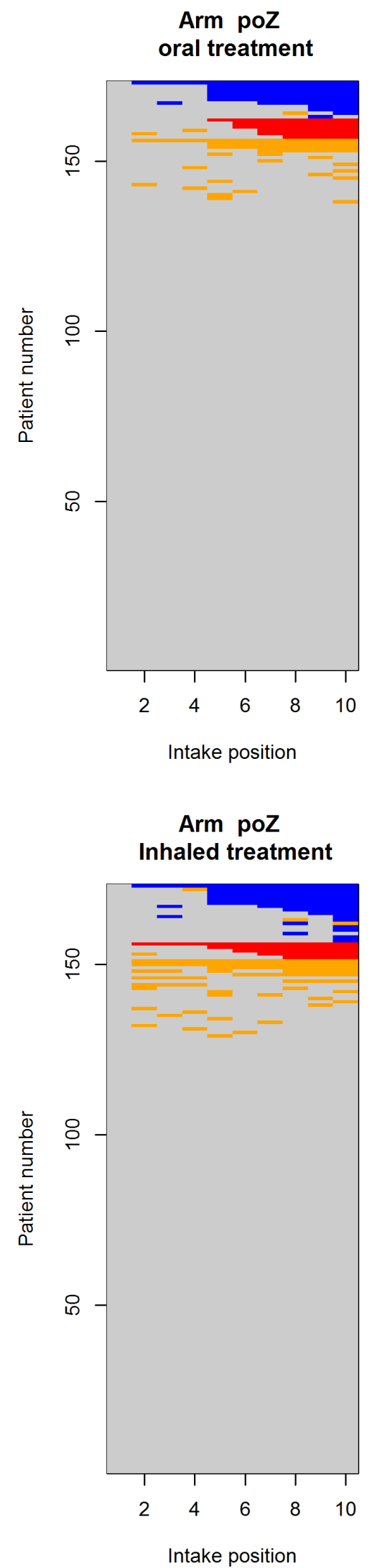
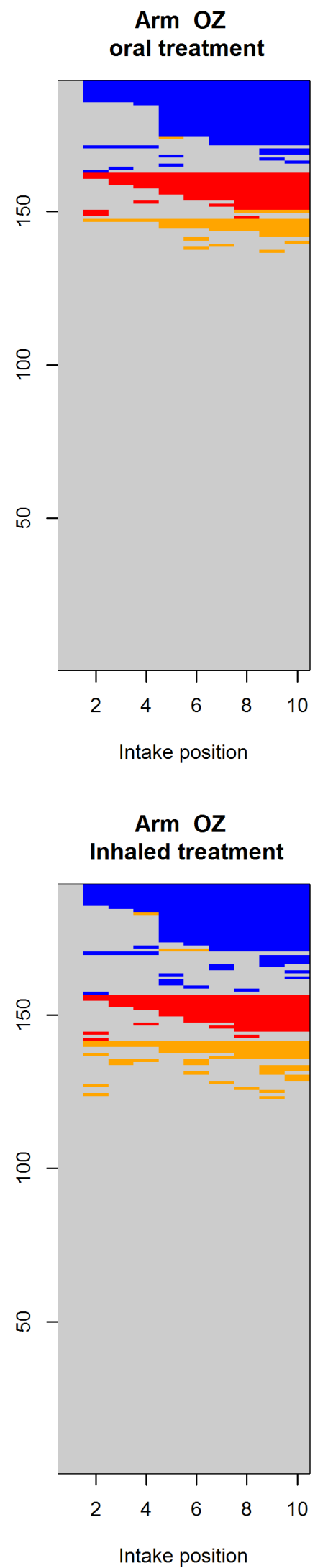
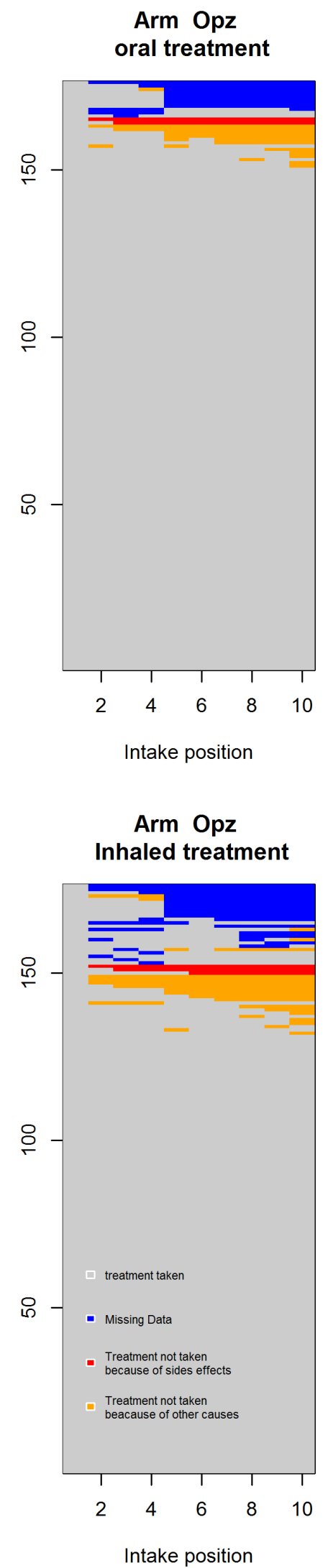

Figure 1 Schematic representation of the 10 medication intakes over the five study days according to the randomisation arm and the route of administration (oral vs inhaled) during the BIVIR trial $(n=541)$. The blue cells correspond to missing data, the red cells correspond to treatment discontinuation due to side effects, and the yellow cells correspond to treatment discontinuation due to other causes (including unspecified causes). 


\section{Kaplan-Meier persistence curves by $\mathbf{4}$ hypothesis of sensitivity analysis}

\section{Persistence curve for oral treatment}
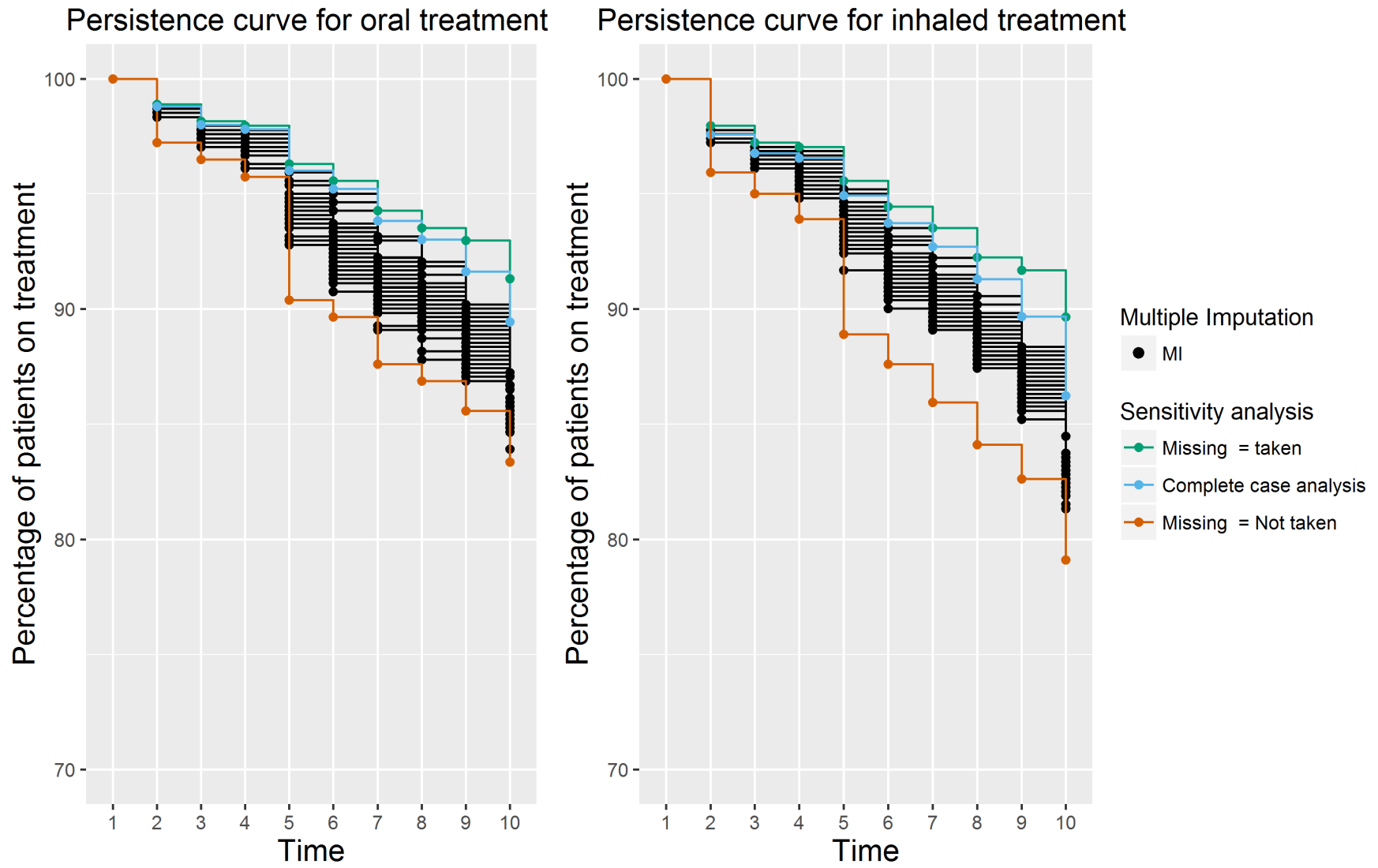

Figure 2 Kaplan-Meier curves for persistence on treatment for oral and inhaled route of administration in BIVIR trial.

and 1.58 ( (95\% CI 1.00 to 2.51$), \mathrm{p}=0.030)$ for the inhaled route.

Multivariate Cox models were then applied. Regardless of the administration route, non-persistence was jointly associated with PCR confirmation of influenza, antibiotic prescriptions and allocation to the $\mathrm{OZ}$ randomisation arm.

Based on the Cox pooled estimates, we found that early dropout was significantly associated with the inhaled route $(\mathrm{HR}=1.23$ (95\% CI 1.01 to 1.49$), \mathrm{p}=0.043)$. No effect of disease score on the hazard of non-persistence was found (HR=0.99 (95\% CI 0.94 to 1.06), $\mathrm{p}=0.34$ ).

The sensitivity analyses (table 2A,B) confirmed that complete case analysis would mask some associations. Note that MICE and the simple imputation of missing data by omission achieved very similar results.

Figure 3 describes the main reason given by patient, nurse or physician to explain non-persistence. The most prevalent cause of non-persistence was side effects, although information on underlying cause was unknown in one-third of the patients. This is otherwise displayed in figure 1: compared with the other arms, there was increased non-persistence in the active biotherapy $(\mathrm{OZ})$ arm due to side effects.

Last, with MICE data sets, we calculated which patients recovered at the time of discontinuation (non-persistent patients). As shown in figure 4, the proportion of recovered patients at the time of discontinuation increased over time from time 6 to time 10 , by which time most of the patients who discontinued their therapies had recovered.

Finally, the results of the multivariable models based on a discrete scale were slightly modified (see online supplementary materials).

\section{DISCUSSION}

Based on the data collected in a double-blind RCT that evaluated the benefits of bitherapy against seasonal influenza, this study found that patient non-persistence increased for the inhaled treatment compared with the oral treatment and for the combination of two active drugs compared with each drug combined with a placebo; in contrast, the virological confirmation of the influenza diagnosis was associated with a longer persistence on treatment.

Despite some restrictions discussed further, the precise description of longitudinal treatment dropout and loss of sight, although rarely detailed, is of interest in RCTs that assess the efficacy of antiviral drugs for treating influenza.

Increased non-adherence to inhaled medications compared with oral medications has already been described, mainly in studies of chronic treatment of patients with asthma. ${ }^{12-14}$ Non-adherence to nebulised antibiotics has also been reported in patients with cystic fibrosis. ${ }^{15}$ However, to our knowledge, this study is the first 


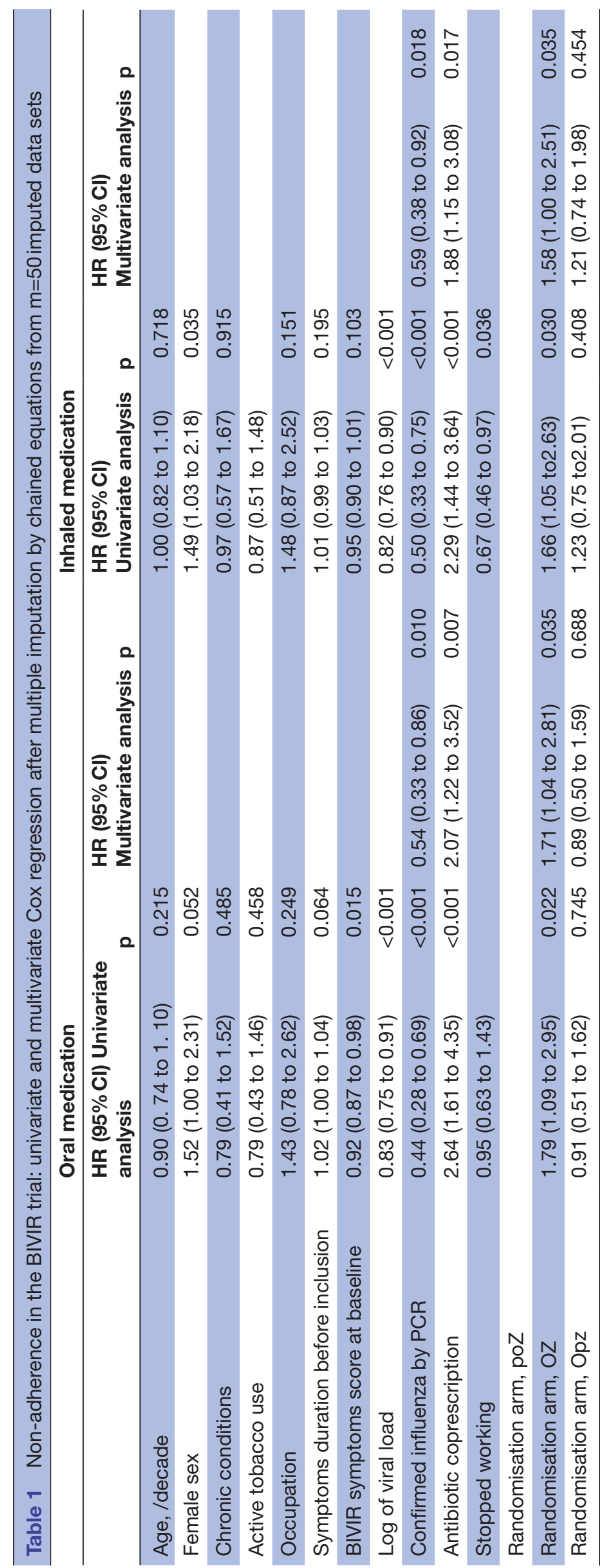

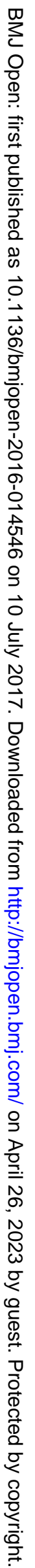




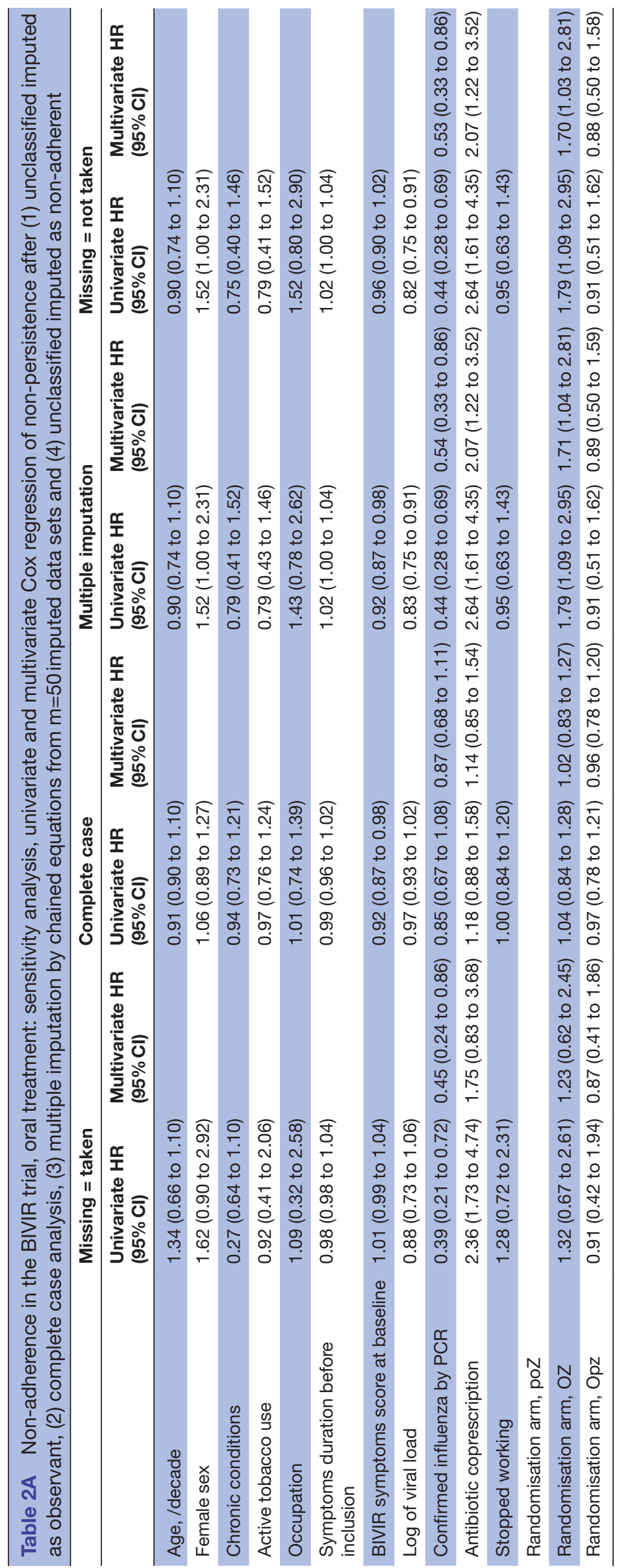




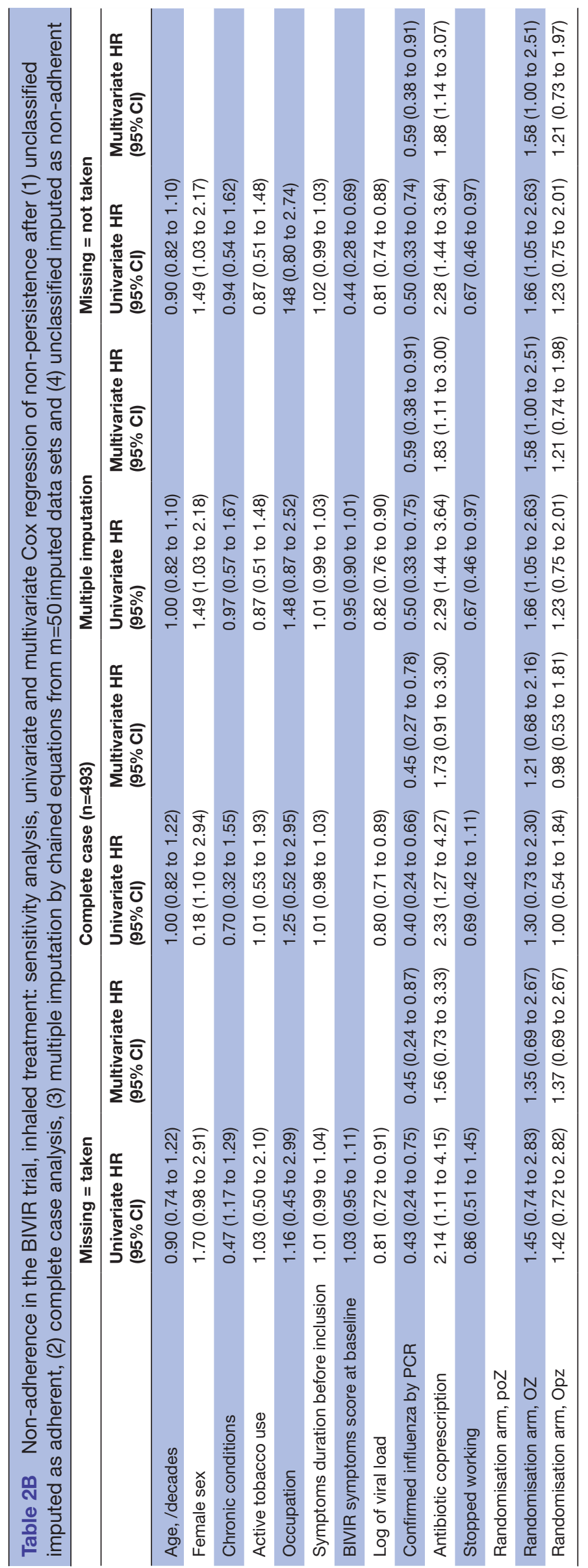




\section{Declared reasons of treatment discontinuation at each time}

\section{Oral treatment}

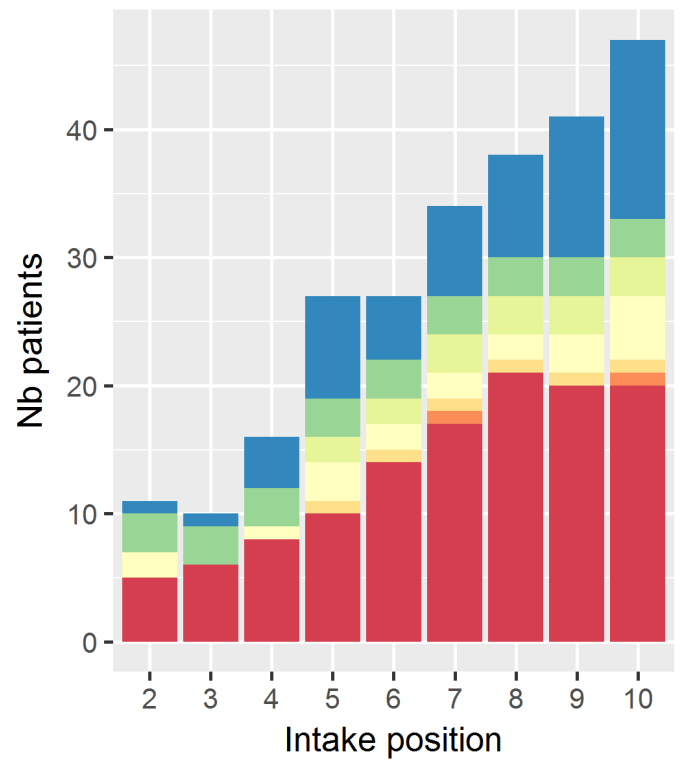

Inhaled treatment

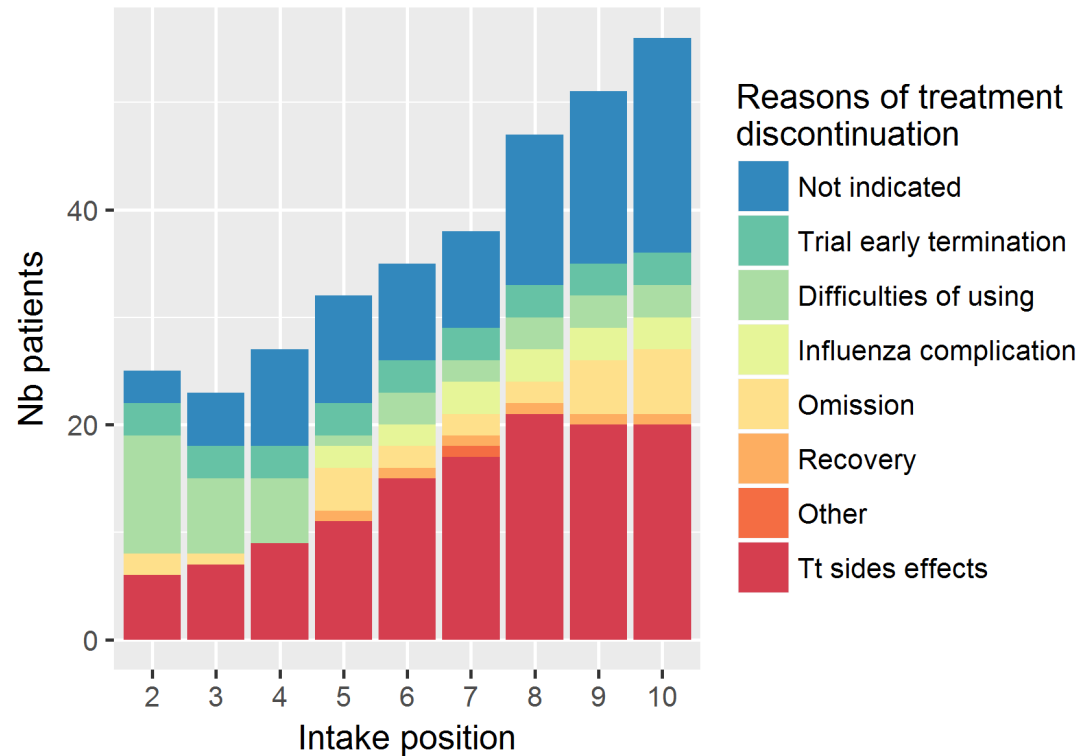

Figure 3 The main reasons for treatment withdrawal, reported by the patients or recorded by the nurses or physicians over the 10 medication intakes during the 5 days of the BIVIR trial.

to report such results for an acute antiviral treatment. In our study, only a few patients reported difficulties using the Diskhaler for the delivery of the inhaled drug; this low prevalence suggests that technical difficulties were not the main reason for the discontinuation of the inhaled treatment. In addition to factors such as the complexity of the inhalation regimen and the patients' understanding of the device and its instructions, treatment non-adherence can be influenced by a number of individual-level factors, including health beliefs, sociocultural and psychological factors and disease severity. ${ }^{14}$ Thus, physicians should be aware of these issues to limit non-adherence. Although the use of drug association may appear difficult to promote in outpatients, its use in critically ill patients could have been of interest if its benefit had been demonstrated. Nevertheless, the strategy of using associations can be jeopardised by non-adherence, while it can only be effective if patients are adherent to both drugs. Non-adherence allows viral replication to take place due to the low drug concentration, which in turn favours the emergence of resistance and subsequent treatment inefficacy. However, whether non-adherence results in inefficacy or inefficacy increases non-adherence remains a matter of debate.

We found that side effects were the most commonly reported cause of treatment discontinuation. This correlation is well known, particularly for psychiatric treatments, where persistence to long-term treatment may be compromised by unpleasant side effects. ${ }^{16}$ The occurrence of side effects is likely increased by drug combinations, as has been extensively documented for HIV antiviral treatments. ${ }^{17} 18$ With respect to the use of neuraminidases for preventing or treating influenza, a recent Cochrane review found that side effects (ie, nausea, vomiting, headaches and renal and psychiatric events) were correlated with oseltamivir use and that patients reported better tolerance for zanamivir. ${ }^{1}$ The reported impact of these side effects on treatment course varies in the literature. ${ }^{19}{ }^{20} \mathrm{~A}$ recent placebo-controlled safety trial of the use of oseltamivir and zanamivir as preventive monotherapies among healthcare workers did not show any difference in drug-related study withdrawals between the treatment and placebo arms. ${ }^{21}$ A recent meta-analysis also reported equal withdrawal rates between the oseltamivir and placebo groups. ${ }^{22}$ In the present study, the patients who were randomised to the active combination treatment arm were more likely to report side effects than the patients who were allocated to the two placebo-containing arms. Moreover, as previously reported, ${ }^{23}$ trial participants who experience unpleasant side effects are less likely to continue treatment than those who have been assured of the efficacy of their treatment.

Despite our efforts to reduce the amount of missing data (see online supplementary materials), $10.3 \%$ of the individual treatment intake data points were missing due to loss of sight. Missing data is a very frequent issue in clinical trials and is often underestimated, despite its strong impact on the measure of treatment efficacy, and is commonly treated by analysing only the available data (complete case analysis). To face this issue, we used MICE imputation, a valid and robust approach that has been well described in the context of clinical trials. ${ }^{24}$ Sensibility analyses show differences between complete case analysis and MICE imputation, while the results of MICE were close to those simply imputing omissions on missing data. Indeed, the patterns of missing data and non-persistence were rather similar, and one could expect that there is 


\section{IIIness status at the time of discontinuation}

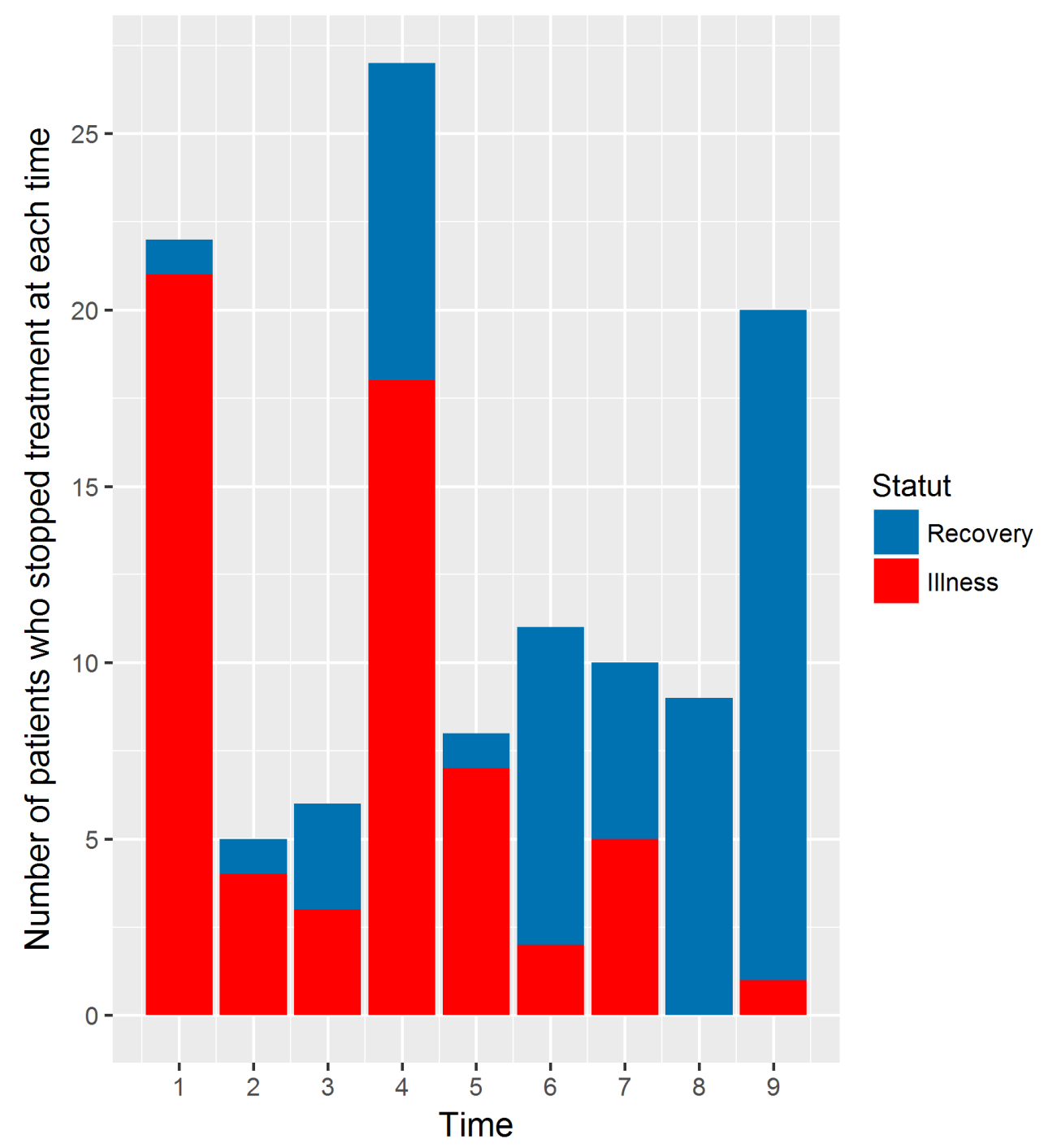

Figure 4 IIIness status at the time of discontinuation over the 10 medication intakes during the 5 days of the BIVIR trial.

a correlation between non-adherence to the research protocol itself (missing data) and to the treatment prescribed (non-adherence to medication). The similarity of these results suggests that modelling by MI could capture a part of this assumption regarding missingness.

Some limitations of our study need to be acknowledged. First, the measure of medication intakes in the BIVR study protocol, using patient diaries, has been shown to overestimate theses intakes due to desirability bias. As described, ${ }^{3}$ the use of electronic medication-event monitoring would have been more appropriate for monitoring medicine intakes. However, the use of complementary sources of information, in particular the PC and the CRF from the GP and nurses, allowed us to correct a large amount of self-reported deviations from prescribed medication and missing data. Combining sources of information is known to be an effective way to improve data quality while studying patient treatment omissions and discontinuation. ${ }^{25}$ This fact does not challenge the association observed between non-persistence, treatment arm and other covariates. Indeed, the strength of these associations would have been underestimated by an overestimation of treatment intakes. Second, some level of residual collinearity between predictors of non-persistence could have persisted in the regression model, notably between the antibiotic coprescription and PCR confirmation. However, we thought they provide extra useful information. Nevertheless, in stepwise regression procedure, both variables remained in the model, suggesting that highly correlated and/or redundant predictors have been removed from the model. Third, we used Cox models, ignoring the discrete scale of our data; we reran analyses with discrete time versions of the proportional hazard model with either a logit link or a c-log-log link, without markedly modifying the results. Last, we used MICE to address the issue of missing data, given it has been a reported method of choice for complex incomplete data problems, and always better than case deletion or single ad-hoc methods, with a great flexibility. ${ }^{10}$ However, resulting estimates are valid under a missing at random 
mechanism, which assumes that the missing treatment intake data were only related to the observed patient characteristics. This assumption could be violated if drug discontinuation and the missing data on drug intake were linked through an unobservable mechanism. Selection models and pattern models have been proposed in this later case, but such models rely on assumptions that have been reported more tenuous than the missing at random mechanism so that a well-done MICE analysis appeared preferable. ${ }^{26}$ However, it is worth noting that the inclusion of appropriate auxiliary variables into the imputation model can go a long way towards reducing the likelihood of violation. The best auxiliary variables are those that are highly correlated with both the variable that has missing data and the probability that the variable is missing. Thus, as recommended, we include as much data as possible in the imputation model.

Moreover in longitudinal studies where the mechanism of missingness is rather dependent on history, MI gives good results because it uses a sequential mechanism for imputation that uses all of the information available at time $\mathrm{t}-1$ to impute missing data at time t.

Finally, we used a common approach in such cases, that is, to perform a sensitivity analysis, by drawing inferences based on a variety of assumptions regarding the missing data mechanism.

Our results suggested that the association between the non-persistence and the allocation to the active bitherapy may question the lack of demonstrated association between that arm and the efficacy provided by the primary intent-totreat analysis. Further analysis, including adherence-based analytical techniques ${ }^{27}$ per protocol, instrumental variables or complier average causal effects analyses, should be required to go further. Nevertheless, such analyses are jeopardised by the fact that, after treatment discontinuation, patients are likely to have been lost to follow-up or withdrawn from the trial, and thus there are only a few data to infer about this issue.

\section{CONCLUSION}

Non-adherence is a major concern in clinical trials given that it can interfere with the interpretation of results, especially in intent-to-treat analyses. We studied patient non-adherence to bitherapy in a double-blind RCT and found that the main reason for treatment discontinuation was the occurrence of side effects. Compared with the two placebo-containing treatment arms, patient non-adherence was higher in the active bitherapy treatment arm. Further analyses should investigate the potential impacts of these findings in evaluating the activity of oseltamivir and zanamivir bitherapy in the BIVIR trial.

\footnotetext{
Author affiliations

${ }^{1}$ IAME (Infection, Antimicrobien, Modélisation, Evolution), UMR-1137, Inserm, Université Paris Diderot, Sorbonne Paris Cite, Paris, France

${ }^{2}$ Service de Biostatistique et Information Médicale, ECSTRA Team, UMR-1153, Inserm, Université Paris Diderot, Sorbonne Paris Cité, Hôpital Saint Louis, Paris, France
}

${ }^{3}$ Aix Marseille Univ, INSERM, IRD, SESSTIM, Sciences Economiques \& Sociales de la Santé \& Traitement de l'Information Médicale, Marseille, France., Marseille, France ${ }^{4}$ ORS PACA, Observatoire régional de la santé Provence-Alpes-Côte d'Azur, Marseille, France., Marseille, France

${ }^{5}$ Faculte de Pharmacie, Université Paris Descartes, Paris, France

${ }^{6}$ Faculté de Médecine, Inserm Université Pierre et Marie Curie, U1136 - Institut Pierre Louis d'épidémiologie et de santé publique (IPLESP), Paris, France ${ }^{7}$ Institut Pasteur, Centre National de Référence des virus influenzae (Région-Nord), Unité de Génétique Moléculaire des Virus à ARN, Paris, France

${ }^{8}$ Réseau des Groupes Régionaux d'Observation de la Grippe (GROG), Coordination Nationale, Paris, France

${ }^{9}$ Faculté de Médecine Lyon Est, VirPatH, EA 4610, Université Claude Bernard Lyon 1, Lyon, France

${ }^{10}$ Laboratoire de Virologie \& CNR des virus influenzae (site Lyon), Hospices Civils de Lyon, Lyon, France

Contributors ICMJE criteria for authorship read and met: CP, AT, TB SVDW, XD, $\mathrm{AM}, \mathrm{CCO}, \mathrm{BL}, \mathrm{CL}$ and $\mathrm{SC}$. Agree with the manuscript's results and conclusions: RF, CP, AT, TB, SVDW, XD, AM, CCO, BL, CL and SC. Designed the experiments/the study: RF, XD, SVDW, CL and SC. Analysed the data: RF, CP, CL and SC. Collected data/did experiments for the study: RF, XD, SVDW, TB, CCO, AT and CL. Enrolled patients: $\mathrm{CCO}$. Wrote the first draft of the paper: RF, CL and SC. Contributed to the writing of the paper: RF, TB, SVDW, BL, CL and SC. Enrolled investigators and took part in the epidemiological surveillance which gave the go-ahead to the study: AM. Responsible for data monitoring: RF. Obtained funding: AM,TB and CL.

Funding This work was supported by a research grant from the French Ministry of Health. The sponsor was Département à la Recherche Clinique et au Développement, Assistance Publique Hôpitaux de Paris (Programme hospitalier de recherche clinique, AOM 06060 and AOM 08209). The funder had no role in study design, data collection and analysis, decision to publish, or preparation of the manuscript.

Competing interests XD has had a conference invitation from GSK and lecture fees from Roche and Gilead. SVDW has had a conference invitation from GSK, research grant from GSK on unrelated subject, joined patent from institution with GSK on unrelated subject, travel grants for meetings from GSK, contribution to clinical trial financed by Roche, member of the advisory committee on influenza of the French Ministry of Health, is a member of ESWI, is a member of the scientific committee of the GEIG and is vice-president of the GROG network. AM has membership in the ministry of health advisory board on influenza, involvement in some epidemiological studies partially or fully granted by Roche and GSK, and travel grants from Roche for participation in scientific meetings. AM received fees from Roche, preclinical pharmacokinetic department, for a course on MONOLIX in December 2008. BL has had paid consultancy and board membership (Roche, GSK, Novartis, BioCryst, Medlmmune), has had research grants from Roche and SanofiPasteur, and had received travel grants and honoraria for speaking or participation at meetings (Roche, Sanofi-Pasteur).

Patient consent Obtained.

Ethics approval Ethics Committee of lle de France 1.

Provenance and peer review Not commissioned; externally peer reviewed.

Data sharing statement Technical appendix, statistical code and data set available on demand to the corresponding author.

Open Access This is an Open Access article distributed in accordance with the Creative Commons Attribution Non Commercial (CC BY-NC 4.0) license, which permits others to distribute, remix, adapt, build upon this work non-commercially, and license their derivative works on different terms, provided the original work is properly cited and the use is non-commercial. See: http://creativecommons.org/ licenses/by-nc/4.0/

(C) Article author(s) (or their employer(s) unless otherwise stated in the text of the article) 2017. All rights reserved. No commercial use is permitted unless otherwise expressly granted.

\section{REFERENCES}

1. Jefferson T, Jones MA, Doshi P, et al. Neuraminidase inhibitors for preventing and treating influenza in healthy adults and children. Cochrane Database Syst Rev 2014:CD008965. CD008965. 
2. Duval X, van der Werf S, Blanchon T, et al. Bivir Study Group. Efficacy of oseltamivir-zanamivir combination compared to each monotherapy for seasonal influenza: a randomized placebocontrolled trial. PLoS Med 2010;7:e1000362.

3. Vrijens B, De Geest S, Hughes DA, et al. A new taxonomy for describing and defining adherence to medications. $\mathrm{Br} J \mathrm{Clin}$ Pharmacol 2012;73:691-705.

4. WHO. Adherence to Long-term therapies: evidence for action. 2003 http://www.who.int/chp/knowledge/publications/adherence_report/ en/ (accessed on Jun 2016).

5. Biomedicine SK. 'nonadherence': a bitter pill for drug trials. Science 2014;346:288-9.

6. Heneghan CJ, Onakpoya I, Jones MA, et al. Neuraminidase inhibitors for influenza: a systematic review and meta-analysis of regulatory and mortality data. Health Technol Assess 2016;20:1-242.

7. Hernán MA, Hernández-Díaz S. Beyond the intention-to-treat in comparative effectiveness research. Clin Trials 2012;9:48-55.

8. Shrier I, Steele RJ, Verhagen E, et al. Beyond intention to treat: what is the right question? Clin Trials 2014;11:28-37.

9. Frangakis C, Rubin DB. Addressing complications of intention-totreat analysis in the combined presence of all-or-none treatmentnoncompliance and subsequent missing outcomes. Biometrika 1999;86:365-79.

10. White IR, Royston P, Wood AM. Multiple imputation using chained equations: Issues and guidance for practice. Stat Med 2011;30:377-99.

11. Buuren S, Groothuis-Oudshoorn K. Mice : Multivariate Imputation by Chained Equations in R. J Stat Softw 2011;45:1-67.

12. Kelloway JS, Wyatt RA, Adlis SA. Comparison of patients' compliance with prescribed oral and inhaled asthma medications. Arch Intern Med 1994;154:1349-52.

13. Cohn RC. A review of the effects of medication delivery systems on treatment adherence in children with asthma. Curr Ther Res Clin Exp 2003;64:34-44.

14. Rau JL. Determinants of patient adherence to an aerosol regimen. Respir Care 2005;50:1346-1359.

15. Latchford G, Duff A, Quinn J, et al. Adherence to nebulised antibiotics in cystic fibrosis. Patient Educ Couns 2009;75:141-4.
16. Mago R, Borra D, Mahajan R. Role of adverse effects in medication nonadherence in bipolar disorder. Harv Rev Psychiatry 2014;22:363-6.

17. Carrieri MP, Leport C, Protopopescu C, et al. Factors associated with nonadherence to highly active antiretroviral therapy: a 5-year followup analysis with correction for the bias induced by missing data in the treatment maintenance phase. J Acquir Immune Defic Syndr 2006;41:477-85.

18. Protopopescu C, Raffi F, Roux P, et al. Factors associated with nonadherence to long-term highly active antiretroviral therapy: a 10 year follow-up analysis with correction for the bias induced by missing data. J Antimicrob Chemother 2009;64:599-606.

19. Strong M, Burrows J, Stedman E, et al; Adverse drug effects following oseltamivir mass treatment and prophylaxis in a school outbreak of 2009 pandemic influenza A(H1N1) in June 2009. 15. Sheffield, United Kingdom: Euro Surveill, 2010. pii/19565.

20. Lee VJ, Yap J, Cook AR, et al. Oseltamivir ring prophylaxis for containment of $2009 \mathrm{H} 1 \mathrm{~N} 1$ influenza outbreaks. N Engl J Med 2010;362:2166-74.

21. Anekthananon T, Pukrittayakamee S, Ratanasuwan W, et al. Oseltamivir and inhaled zanamivir as influenza prophylaxis in Thai health workers: a randomized, double-blind, placebocontrolled safety trial over 16 weeks. J Antimicrob Chemother 2013;68:697-707.

22. Dobson J, Whitley RJ, Pocock S, et al. Oseltamivir treatment for influenza in adults: a meta-analysis of randomised controlled trials. Lancet 2015;385:1729-37.

23. Dodd S, White IR, Williamson P. Nonadherence to treatment protocol in published randomised controlled trials: a review. Trials 2012;13:84.

24. Panel on Handling Missing Data in clinical trials national research council of the national academies, the prevention and treatment of missing data in clinical trials., the national academies Press, 2010.

25. Liu H, Golin CE, Miller LG, et al. A comparison study of multiple measures of adherence to HIV protease inhibitors. Ann Intern Med 2001;134:968-77.

26. Enders CK. Applied Missing Data analysis. New York, 2010.

27. Ye C, Beyene J, Browne G, et al. Estimating treatment effects in randomised controlled trials with non-compliance: a simulation study. BMJ Open 2014;4:e005362. 